\title{
Elijah High Altitude Balloon Launch Team 2012-2013
}

Tyler Capek, University of Wisconsin River Falls; Richard Oliphant, Milwaukee School of

Engineering; Devin Turner, Marquette University; Danielle Weiland, Carthage College

\section{Wisconsin Space Grant Consortium}

\begin{abstract}
The 2012-2013 launch team of four undergraduate students has brought itself up to speed on the equipment and software necessary for high altitude balloon flights, prepared and tested it all, and completed two successful flights. GPS flight data of latitude, longitude, time, and altitude was collected and analyzed. A new mobile tracking tool was used more than ever before with the growing popularity of smart phones; and new methods were used for data analysis and presentation thanks to technologies now available on the internet.
\end{abstract}

\section{Introduction}

The Student Satellite Initiate is an innovative program that provides students with the opportunity to fly their science experiments in a near space environment. The Elijah high altitude balloon launch team is funded by the Wisconsin Space Grant Consortium (WSGC) to work together to safely launch and return a scientific payload for data analysis. The launch team is tasked with assisting in the launch of the balloons as well as tracking the balloon while it is in the air. The launch team is also responsible for the maintaining and updating of the tracking payload. Data retrieved from the tracking payload is used to show the path of the balloon. Given the nature of the task and equipment, specialized procedures and coordination within the team are required, as well as equipment testing and accurate selection of a suitable launch location. The Elijah launch team has performed two successful launches and recoveries of the high-altitude balloon and payload and has more launches planned in upcoming months.

\section{Equipment and Testing}

The Federal Aviation Administration (FAA) allows two payloads to fly with a high altitude balloon: a six-pound payload and a two-pound payload. The six-pound payload is left to the Elijah Payload Team, and the two-pound payload is made up of the launch team's positioning system.

Before launch day the necessary equipment must be gathered and tested. The following is a list of the equipment needed with a brief explanation of each item:

- Helium - a primary tank and a secondary tank

- Balloon - these natural latex balloons range from 800 to 3,000 grams

- Tarp - used as a ground cover for a clean surface to inflate the balloon on

- Gloves - the latex balloon will rupture earlier if contaminated by skin oils

- O-ring - clamps around the balloon collar for efficient filling

- Air hose - hosing from the helium tank to the balloon collar

- Parachute - attached below the balloon but above the payloads, this slows the descent rate of the system after the balloon bursts

- Tracking system - two GPS modules for tracking 
- Science payload - maximum of six pounds, this module is mounted last

- Scale - used to confirm weight regulations are met before launch

- Counterweight - adjustable weight to test balloon lift before launch

- Quick release nozzle - connection between helium hose and balloon collar

Several systems mandate preceding testing to ensure accuracy and functionality for launch operations. In the week leading up to the launch date, the GPS transmitter with the StratoSat tracking software was tested on the ground in Milwaukee. A three hour test was run and all systems proved operational. In the 24 hours before launch, each module's electrical pack was charged as well.

\section{Pre-launch Operations}

In addition to an equipment check and testing, the launch team is responsible for finding a suitable launch date and location. Near Space Ventures makes available a website which takes certain inputs and generates a flight path prediction for high altitude balloons(Campbell, 2012). The inputs include the launch date and time, the launch site location (longitude \& latitude) and elevation, an appropriate weather station, the anticipated balloon ascent and descent rates, as well as its anticipated burst altitude (Figure 1).

Launch date selection. High altitude balloons are rather fragile and necessitate calm weather. When discussing potential launch dates, the weather forecast is evaluated. Once the weather is confirmed by several sources to be forecast as calm, the jet stream is evaluated. Balloon flights should not pass through a turbulent or violent jet stream. Again, the balloon, payload, and tracking equipment are somewhat fragile and need to stay in predictable environments. A quick check to California Regional Weather Service's website gives a map of the jet stream across North America and shows grayed-out zones where the jet stream is moving rapidly and violently. This can be forecasted five days in advance.

Weather station selection. It is best to use a weather station that is in the middle of the predicted flight path, the Near Space Ventures application uses weather and wind data from the National Weather Service's forecast soundings from the specified station to predict the behavior of the balloon. The three letter airport code is all that is needed to specify a station.

Launch site selection. When selecting launch sites, a large public field away from tall trees, overhead wires/cables, and air traffic (airports) is necessary. It is preferable to have a wi-fi hotspot nearby for internet access for running one or two last predictions. This is usually found at a café or restaurant so food is there for the launch team too. If a team is fatigued, bad things could happen. These locations are plentiful however, so more primary factors are used to determine a launch site first. First, a launch site is chosen and the necessary information gathered for it (i.e. latitude, longitude, elevation, and weather station). This is inputted and the Near Space Ventures application outputs a predicted path on Google Maps with a landing site inside a 5 mile radius circle and a 10 mile radius (Figure 2). From this, the predicted landing site is analyzed. Google Maps satellite view is used to look for areas of water, cites or towns, as well as forested or hilly terrain. These are avoided. From the resulting predicted path, the launch team sees where the winds tend to be taking the balloon and this becomes an iterative process. Another location is chosen with the first prediction as a reference, the output is analyzed, and the process repeats 
until a suitable launch and landing combination is obtained. This is done a week prior to the launch date, a few days before the launch date, and then the night before, and the morning of. The weather predictions become more accurate as the launch date approaches and sometimes the launch site needs to be changed.

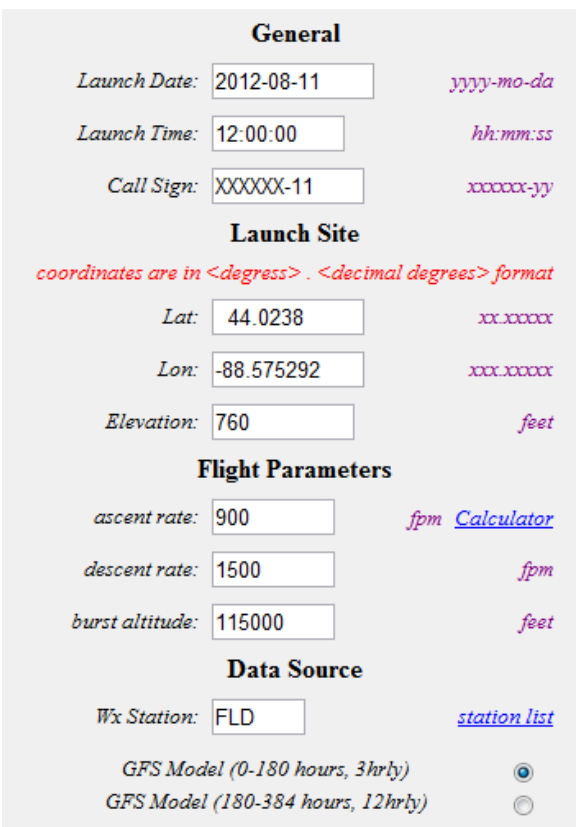

Figure 1: Data required for flight prediction.

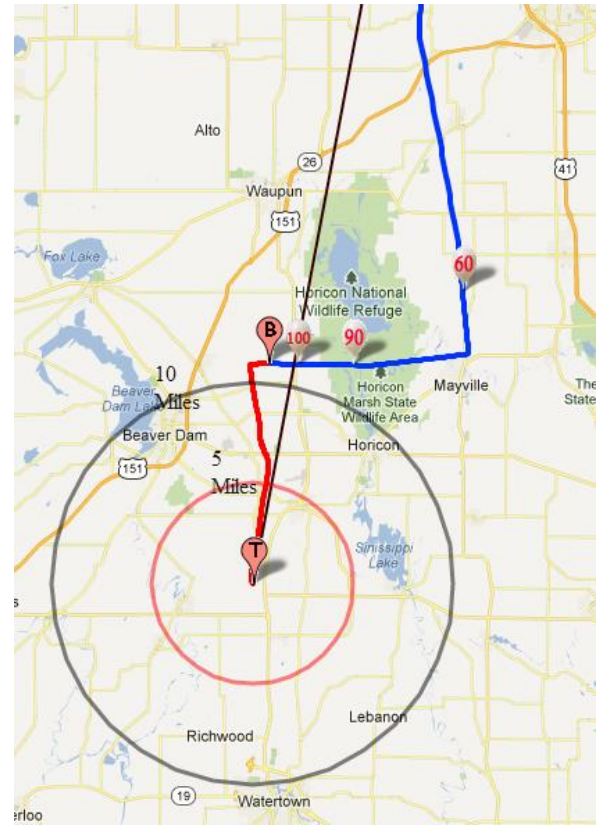

Figure 2: Google map result of flight prediction.

Once a specific area has been determined to be a good launch location, Google earth is used to select a mark. This mark is sent to all of the launch team, payload team, and the team advisor for use as reference on launch day.

\section{Launch Day Operations}

On the day of the launch a flight path prediction is run to confirm all is well for the chosen launch site, path, and landing zone. The equipment is gathered up from the previous day's test and electrical pack charging, and loaded into the team's vehicles.

Once at the launch site with the payload team, the tarp is laid down in an open and level area. From this spot the balloon is prepared by carefully unrolling it onto the tarp by team members wearing gloves. Others hook-up the helium tank to the hose and then to the neck and collar of the balloon for filling. The payload system is also set-up. The parachute is unraveled and then folded so that it will open properly on descent, then the first GPS module is attached below that, then the secondary one, and lastly the science payload. The GPS modules are turned on and the StratoSat software is activated to verify a signal is being transmitted. The science payload is powered on as the balloon is filled with helium. The balloon is not yet connected to the payload system, but instead to the counterweight. The counterweight is set to six pounds heavier than the sum of the weight of the payload system. Then as the balloon fills, it works to lift the counterweight. Once the balloon can lift the counterweight off of the tarp, the helium is shut off, the balloon collar connection to the payload system confirmed, and the quick release nozzle is released. The balloon is let go and as it rises, each successive payload module is released (Figure 
3). Holding the modules by their connecting cord and releasing in succession stops the balloon lift from yanking each payload violently off of the ground.

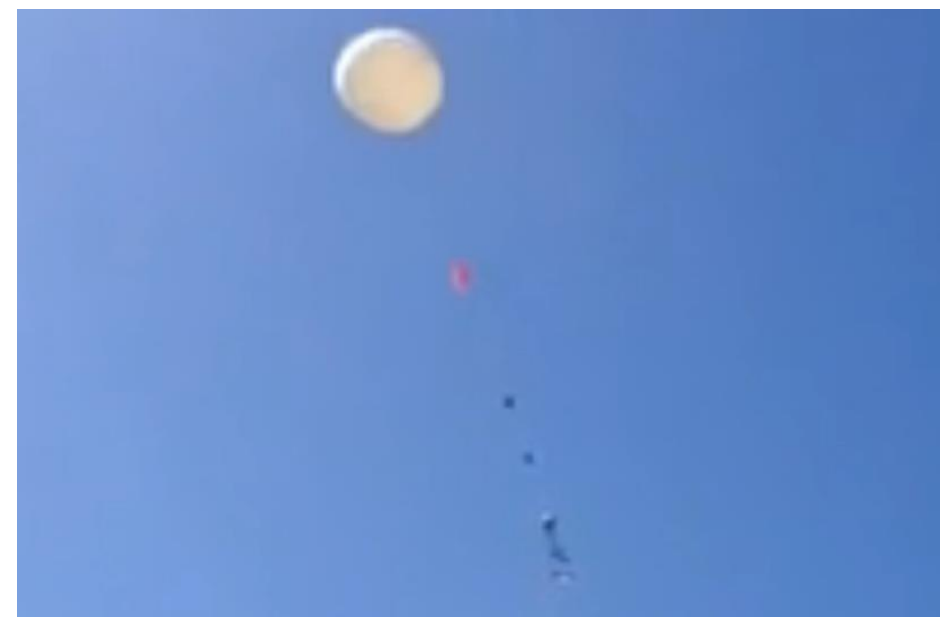

Figure 3: The balloon and payloads immediately after release.

\section{Tracking}

Once in flight, the balloon and payload system soar out of sight and can only be tracked by the GPS signals. The tracking pod pings out a GPS coordinate along with its speed and direction of motion every 30 seconds or so (Czech, Fossen, Johnson, \& Westphal, 2010). The signal is received by antennae secured to the chase vehicles and plotted on a map. Using StratoSat and MapPoint software, the balloon is tracked in real time. This is in addition to the web browser application that plots the balloon system's path and can be accessed by any device with internet access. This tracking application was advertised by the launch and payload teams for a wide community audience. The teams also had a record number of smart phones that could track the balloon's progress as well (Figure 4).

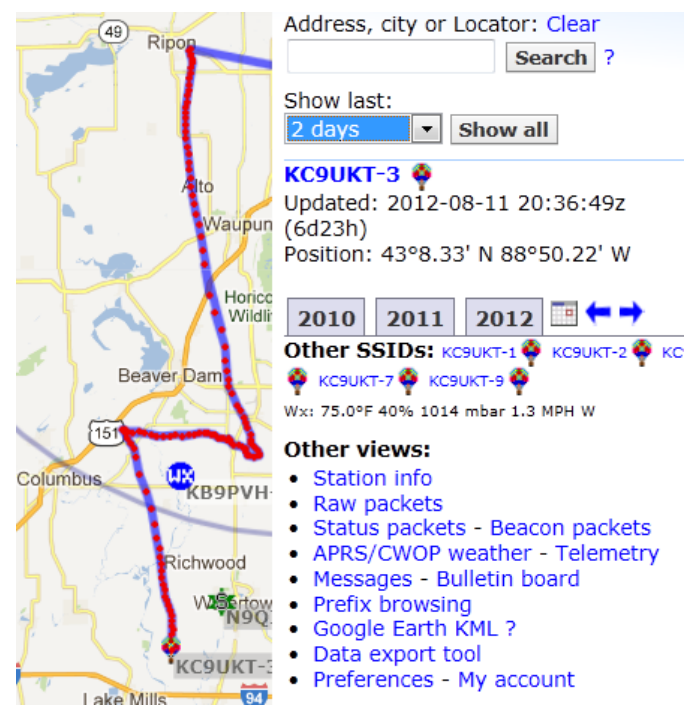

Figure 4: Screen shot of the mobile web app used for tracking. 
The chase vehicles communicate via short range radios and cell phones while driving to the predicted landing zone. As the balloon flies it often drifts from the predicted path, and the chase vehicles must make quick decisions to change their route in order to get to the actual landing site.

\section{Recovery}

The tracking software has a variance of plus or minus 50 feet (Garver \& Krueger, 2009). This allows the launch team to get to the general site of recovering the payload system, but often this isn't enough to find it. If the chase vehicles aren't able to predict the actual landing site accurately or get there in time to see the system descend, or if the system falls into tall vegetation, then it can be difficult to recover.

First launch. On the first launch the balloon landed in an easily accessible location - only 60 feet off a country highway, into two-foot tall corn.

Second launch. On the second launch the location was less accessible and the payload sustained major damage. Upon recovering the payload, it was determined that the payload sustained damage in-flight and parts of the payload were unrecoverable. In-flight damage to balloon payloads has been a challenge historically and is likely a result of severe turbulence experienced by the balloon as it travels through the jet stream during the flight. Payload damage can also be caused during landing but the missing payload items were not found in the area of the landing site. The parachute, balloon throat, GPS transmitters and harnesses were not damaged in landing, suggesting that the damage was not a result of an in-flight collision. The audio tracking component (beeper) that was added to aid in the recovery of the payload significantly improved our ability to locate the payload after landing and will be used in future flights as well.

\section{Flight analysis}

The data received from the tracking payload was loaded into an excel spreadsheet. The geographic coordinate data was outputted in a degree-minute format (DMF, 43 $\left.{ }^{\circ} 48.7235\right)$. In order to work with most programs, the geographic coordinate data had to be converted into a degree-decimal format (DDF, 43.81205833 ${ }^{\circ}$. This was accomplished using the following excel algorithm:

$$
\left(\frac{\mathrm{DMF}-\mathrm{ROUNDDOWN}(\mathrm{DMF},-2)}{60}\right)+\left(\frac{\text { ROUNDDOWN }(\mathrm{DMF},-2)}{100}\right)=D D F
$$

Figure 5: Excel algorithm for converting degree-minute data to degree-decimal data

Altitude data for each geographic coordinate was also isolated. It was outputted in meters, and no conversion was needed. The geographic coordinate and altitude data was exported into an extensible markup language (XML) document. XML is a programming language that defines a set of rules for encoding documents that is readable by both humans and machines. The XML document was needed to convert the data into a format that Google Earth could read. Google Earth is a virtual globe, map, and geographical information program. The XML document was designed to create a path along the geographic coordinate data, essentially tracking the entire path of the balloon's flight. The code was copied and pasted into Google Earth, where the flight of the balloon could be visually depicted as shown in Figures 6-8. 


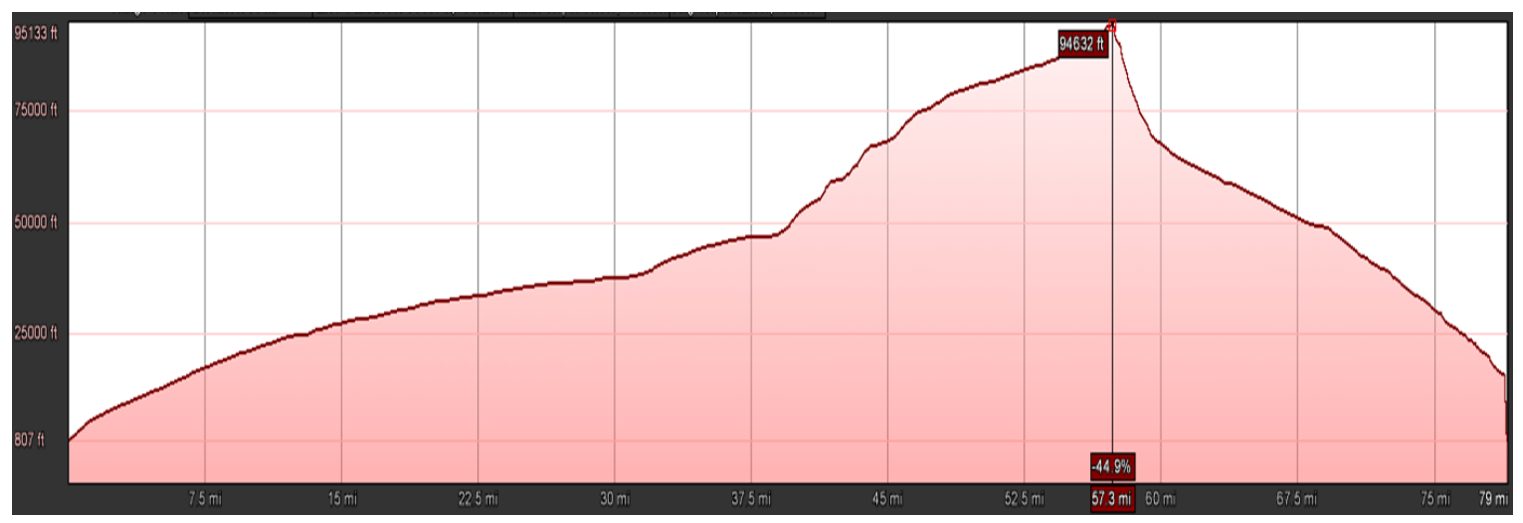

Figure 6: Graph of the altitude with respect to the total distance traveled by the balloon.

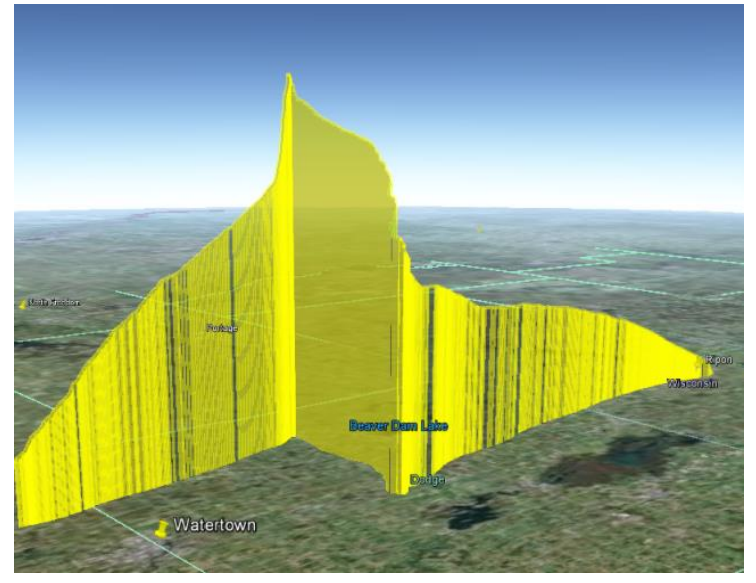

Figure 7: Three dimensional portrayal of the balloon's flight path in Google Earth.

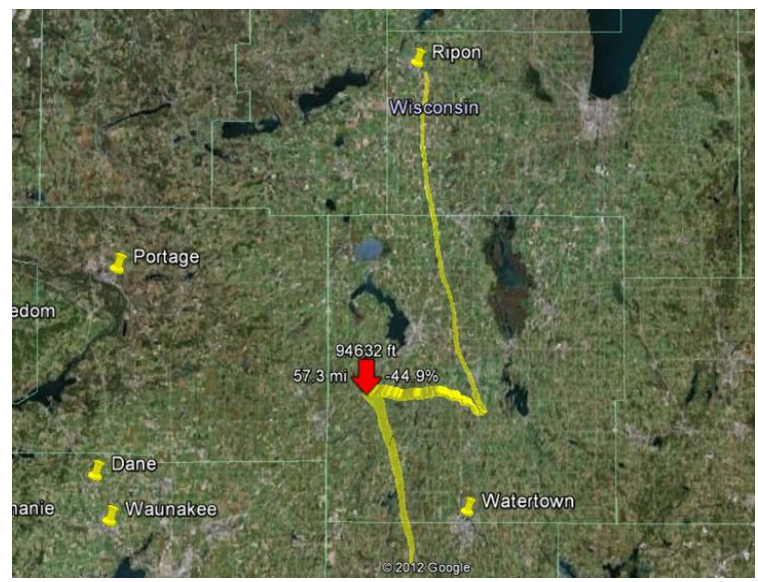

Figure 8: Superior view of the balloon's flight path in Google Earth.

\section{Conclusion}

The team completed two successful launches and recoveries reaching altitudes in excess of 100,000 feet and sustained flight time of over two hours. In addition to learning how to smoothly plan future launches, the team efficiently handled testing, coordination and transportation of the balloon and payload. However upon recovery it was discovered that several parts of the payload had separated from the harness during flight. Some areas of improvement for future launches include a higher fidelity jet stream model, starting the chase phase more quickly, launching earlier in the day, better securing of the payload to the harness, and updating the GPS tracking systems for easier recovery.

\section{References}

1. Campbell, T. (2012, August 15). On-line Near Space Flight Track Prediction Utility. Retrieved from Near Space Ventures, Inc.

2. Czech, M., Fossen, T. V., Johnson, P., \& Westphal, K. (2010). Balloon Tracking Methods. Wisconsin Space Grant Consortium Confererence Proceedings. 21, (pp. 11-17).

3. Garver, M., \& Krueger, J. (2009). StratoSAT Instruction Manual. Stratrostar Systems LLC 


\section{Acknowledgements}

Dr. William Farrow, Milwaukee School of Engineering

Brittany Luedtke and Sharon Brandt, WSGC Office at UW-Green Bay

Rich Phillips, Milwaukee School of Engineering

WSGC Satellite Initiative High Altitude Payload Members 